\title{
44445 - REMOVAL OF CENTRAL VENOUS CATHETERS DUE TO SUSPECTED CATHETER-RELATED INFECTION
}

Santosh Patel, Harborview Medical Center, Seattle, WA, USA;

Finbar O'Neill, Royal victoria Hospital;

INTRODUCTION:

The aim of the present audit was to describe the practice of central venous catheter (CVC) removal and outcomes of suspected catheter-related infection (SCaRI).

METHODS:

Local IRB approval was obtained for this study. Using a structured form, prospectively data were collected over 3 month period in an 18 bedded multidisciplinary ICU. Causes of CVC removal and number of days in situ were noted. Their primary admission diagnosis was noted. The factors contributed to suspicion of catheter related bloodstream infection (CaRBI) including exit site infection, clinical and laboratory parameters were noted. If removed due to SCaRI other proven sources of infection and blood culture results available were recorded. Microbiology follow up was done for all removed catheters in the ICU.

RESULTS:

65 CVCs were removed in 35 patients due to various reasons (figure). 37 (57\%) CVCs from IJV, 15 (23\%) from SV and 13 (20\%) from FV were removed. The shortest and longest in situ period was 2 and 16 days respectively (mean 6.7 days). 50\% of CVCs removed were antiseptic impregnated. SCaRI was the commonest cause of removal $(30 ; 45 \%)$ followed by 'not needed' $(17 ; 25 \%)$. Unresolved pyrexia, increasing vasopressor support and or raised WCC count were the commonest factors contributed towards SCaRI. When removed due to SCaRI, on 70\% occasions one or more proven or strongly suspected causes of sepsis were present. Peripheral blood cultures were positive in only 4 patients when removed due to SCaRI. Of $30 \mathrm{CVCs}$ removed due to SCaRI 18 (60\%) did not reveal any growth, $5(16.6 \%)$ CVCs were colonized with coagulase negative staphylococci (CNS) and 7 (23.4\%) had grown various organisms of which $5(16.6 \%)$ were candida species. 35 (55\%) CVCs were removed due to other reasons apart from SCaRI. Of which 8 (22.9\%) were colonized with CNS, $23(65.4 \%)$ had no growth. 4 CVCs could not be found on microbiology data. All the proven cases of CaRBI were treated with appropriate antibiotics.

DISCUSSION: The question of when removal of CVC is appropriate in the setting of SCaRI presents an important clinical dilemma. While failure to remove the catheter is associated with considerable morbidity, reinsertion is also a procedure associated with both resource consumption and morbidity. Our data suggest that SCaRI was found to be the leading cause of CVC removal. Unfortunately, CVCs removal due to SCaRI was ordered mainly on clinical judgment. The removal of CVC in the presence of other proven infections is a challenging decision and requires experience. An attempt to diagnose CaRBI while catheter is in situ should be made by peripheral and central blood cultures (1). A policy based on evidence (2) 
might decrease the incidence of CVC removal due to SCaRI. A larger audit is planned to develop local guideline.

REFERENCES:

1.N Engl J Med 2003 348: 1123-33.

2.Clin Infect Dis 2001 32: 1249—1272

Reasons for removal of CVCs

\begin{tabular}{|lll|}
\hline ScaRI & $\square$ Not needed & $\square$ Too old \\
$\square$ Malfunction & $\square$ Accidental & $\square$ Malposition \\
$\square$ Change over guidewire & $\square$ Diarritoea & $\square$ Unknown \\
\hline
\end{tabular}

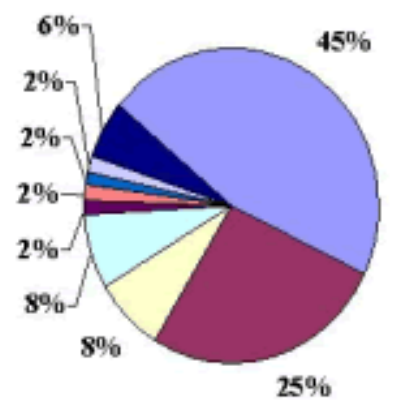

